\title{
Fumonisin $\mathrm{B}_{1}$ and beauvericin accumulation in wheat kernels after seed-borne infection with Fusarium proliferatum
}

\author{
Zhiqing Guo ${ }^{1}$, Katharina Pfohl'2, Petr Karlovsky², Heinz-Wilhelm Dehne ${ }^{1}$, Boran Altincicek ${ }^{1}$ \\ ${ }^{1}$ Institute of Crop Science and Resource Conservation (INRES-Phytomedicine), Rheinische Friedrich-Wilhelms-University of \\ Bonn, Meckenheimer Allee 166a, D-53115 Bonn, Germany \\ ${ }^{2}$ Molecular Phytopathology and Mycotoxin Research, Georg-August-University Göttingen, Grisebachstrasse 6, D-37077 \\ Göttingen, Germany \\ e-mail: altincicek@hotmail.de
}

\begin{abstract}
Fusarium proliferatum is a fungal pathogen causing ear rot of maize. The fungus infects a range of other plants but the economic impact of these diseases has not been established. Recently, F. proliferatum and its mycotoxin fumonisin were found in wheat grains. Here we report that seed-borne infection of wheat with F. proliferatum resulted in systemic colonization of wheat plants and contamination of wheat grains with fumonisins and beauvericin. F. proliferatum strains originating from different hosts were able to infect wheat via seeds. Colonization of wheat plants with the fungus was highest in the stems, followed by leaves; one third of the strains reached kernels, causing accumulation of fumonisins and beauvericin to $15-55 \mu \mathrm{g} \mathrm{kg}^{-1}$. The results show that seed-borne infection of wheat with F. proliferatum can lead to contamination of wheat kernels with mycotoxins fumonisins and beauvericin.
\end{abstract}

Key words: systemic colonization, mycotoxins quantification, fungal DNA, mycotoxins contamination

\section{Introduction}

Fusarium proliferatum is a member of Gibberella fujikuroi species complex which consists of eight mating populations (MPs) and a number of asexual lineages (Leslie et al. 2006), described as morphological species (Nirenberg and O'Donnell 1998). Most members of G. fujikuroi species complex are economically important plant pathogens. Decreased yield as well as diminished quality of plant products due to Fusarium infection cause significant economic losses worldwide (Placinta et al. 1999). F. proliferatum is a member of the complex with a wide host range. The pathogen was predominantly found in maize (Zea mays L.) but it also infects rice (Abbas et al. 1999) and sorghum (Bacon and Nelson 1994) and causes crown, spear and root rot of asparagus (von Bargen et al. 2009), bulb rot in garlic and onion (Stankovic et al. 2007), and fruit rot in date palms (Abdalla et al. 2000).

Grains infected with F. proliferatum accumulate mycotoxins. F. proliferatum and F. verticillioides are the main source contamination with fumonisins, which are toxic and carcinogenic. Fumonisin $B_{1}$ is the most abundant fumonisin found in maize. Fumonisins also can be detected in symptomless infected maize kernels (Bacon and Hinton 1996). These mycotoxins inhibit sphinganine/sphingosine $\mathrm{N}$-acyltransferase (ceramide synthase), a key enzyme in the sphingolipid metabolism and triggers apoptosis (Riley et al. 1996). High levels of fumonisins in maize were associated with outbreaks of equine leukoencephalomalacia (Wilson et al. 1990) and swine pulmonary edema (Osweiler et al. 1992). Association between fumonisin exposure and esophageal cancer was reported in South Africa (Rheeder et al. 1992). Besides fumonisins, F. proliferatum was shown to synthesize moniliformin (Marasas et al. 1986), beauvericin (Moretti et al. 1994) and fusaproliferin in maize kernel cultures (Ritieni et al. 1995), and fusarins in mineral medium consisting of ultrapure water (1 I), $\left(\mathrm{NH}_{4}\right)_{2} \mathrm{HPO}_{4}(1 \mathrm{~g}), \mathrm{KH}_{2} \mathrm{PO}_{4}(3 \mathrm{~g}), \mathrm{MgSO}_{4} \cdot 7 \mathrm{H}_{2} \mathrm{O}(0.2 \mathrm{~g}), \mathrm{NaCl}(5 \mathrm{~g})$, sucrose (40 g) and glycerol (10 g; final pH 6.2), which was also satisfactory for moniliformin production (Miller et al. 1995). Besides F. proliferatum, other causal agent pathogens of Fusarium head blight synthesize moniliformin, beauvericin, fusarins, and fusaproliferin in grains. Moniliformin is produced by F. culmorum in rice culture (Scott et al. 1987), by F. avenaceum and F. oxysporum in parboiled rice culture (Abbas et al. 1989). Beauvericin is produced by F. avenaceum in wheat (Logrieco et al. 2002) and by F. poae, F. oxysporum (Logrieco et al. 1998) and F. verticillioides (Leslie et al. 2004) in maize kernels. Fusarin C is produced by major causal agents of Fusarium head blight in maize grains, and synthesized by F. avenaceum in parboiled rice culture (Abbas et al. 1989). F. verticillioides can produce trace levels of fusaproliferin in autoclaved yellow maize kernels (Leslie et al. 2004). Fusaproliferin is not produced by any Fusarium species that has been found in wheat except for F. proliferatum. 
Systemic infection of host plants has been reported for several Fusarium species. The entire maize plant can be systemically colonized by F. verticillioides without symptoms. In this way, F. verticillioides can be transmitted via seeds (Munkvold et al. 1997). Bacon and Hinton (1996) reported that maize kernels infected with F. verticillioides (F. moniliforme in their article) served the pathogen as a dissemination vehicle. The fungus grew intercellularly without visual signs of diseases through the whole plant, whereas both intercellular and intracellular hyphae were found in plants with disease symptoms. Plants other than maize can be systemically colonized with F. verticillioides, too (Dastjerdi and Karlovsky 2015).

Infection of wheat kernels with F. proliferatum was reported for the first time from Canada, where the pathogen was found as a rare contaminant of tombstone kernels (Clear and Patrick 1990). In 1992, F. proliferatum was isolated from infected wheat seeds in Mazandaran (Zamani-Zadeh and Forutan 1992). The following study from Canada identified F. proliferatum as the causal agent of black point symptom in wheat (Conner et al. 1996). Spontaneous infection of wheat plants grown in a space shuttle with F. proliferatum (Bishop et al. 1997) motivated a series of physiological studies with little relevance for field pathology but the same laboratory identified $F$. proliferatum as an opportunistic pathogen of wheat under stress conditions (Kwon et al. 2001). A survey of fumonisins determination in cereals in Spain reported fumonisins in 8 of 17 samples and F. proliferatum infecting 2 of 17 samples naturally (Castella et al. 1999). Field pathologists became interested in F. proliferatum in wheat after contamination of wheat kernels with fumonisins was reported from Italy (Cirillo et al. 2003). In spite of a growing number of studies of F. proliferatum in wheat in recent years (Desjardins et al. 2007, Palacios et al. 2011, Busman et al. 2012, Amato et al. 2015), our understanding of this pathosystem is still limited.

The objective of present study was to assess the ability of $F$. proliferatum to colonize wheat plants systemically and to compare aggressiveness of strains isolated from different hosts towards wheat.

\section{Material and methods}

\section{Fungal strains and wheat plants}

Sixteen single spore strains of F. proliferatum isolated from garlic, onion, asparagus, maize, silver grass and a dead larva of Zeuzera pyrina in Germany, France, Syria and Austria were used (Table 1). The strains were taxonomically assigned to F. proliferatum based on micro-morphological features on carnation leaf agar (CLA) (Leslie et al. 2006) and the taxonomical identification was confirmed by species-specific PCR with primers PRO1 and PRO2 (Mulè et al. 2004).

Table 1. F. proliferatum strains

\begin{tabular}{|c|c|c|c|}
\hline Strains $^{\mathrm{aT}}$ & Host & Year & Origin \\
\hline F. pro 2-G & Garlic & 2000 & France \\
\hline F. pro 67-S & Silver grass & 1993 & Brandenburg, Germany \\
\hline F. pro 78-S & Silver grass & 1993 & Brandenburg, Germany \\
\hline F. pro 86-S & Silver grass & 1993 & Brandenburg, Germany \\
\hline F. pro 2-O & Onion & 2008 & Baden-Wuerttemberg, Germany \\
\hline F. pro 5-O & Onion & 2008 & Rheinland-Pfalz, Germany \\
\hline F. pro 29-M & Maize & 2005 & Brandenburg, Germany \\
\hline F. pro 76-M & Maize & 2008 & Baden-W. Germany \\
\hline F. pro 219-A & Asparagus & 2000 & Rheinland-Pfalz, Germany \\
\hline F. pro 223-A & Asparagus & 1997 & Brandenburg, Germany \\
\hline F. pro 227-A & Asparagus & 2003 & Goldgeben, Lower Austria \\
\hline F. pro 231-A & Asparagus & 2003 & Goldgeben, Lower Austria \\
\hline F. pro 241-A & Asparagus & 2003 & Upper Austria \\
\hline F. pro 245-A & Asparagus & 2003 & Burgenland, Austria \\
\hline F. pro 259-A & Asparagus & 2003 & Lower Austria \\
\hline F. pro 3-L & Insect Larva & 2005 & Syria \\
\hline
\end{tabular}


Bread wheat (Triticum aestivum L.) cultivar "Taifun" (KWS, Germany) was grown under greenhouse conditions. The cultivar is rated as susceptible to Fusarium infection (score 6 on German scale from 1 [resistant] to 9 [susceptible], Anonymous 2014).

\section{Preparation of inocula}

Mung bean medium according to Bai and Shaner (1996) in $50 \mathrm{ml}$ portions in $100 \mathrm{ml}$ Erlenmeyer flasks was autoclaved at $121^{\circ} \mathrm{C}$ for $20 \mathrm{~min}$. Three discs of $5 \mathrm{~mm}$ diameter from potato dextrose agar (PDA) plates overgrown with F. proliferatum were inoculated into each flask and incubated at $25{ }^{\circ} \mathrm{C}$ without light for 4 days with shaking at $150 \mathrm{rpm}$. Using four-layer cheese cloth for filtering the mycelia, conidia were collected, enumerated in a haemocytometer cell and stored in $-80^{\circ} \mathrm{C}$.

\section{Inoculation of wheat plants}

Wheat seeds were surface-disinfested for $3 \mathrm{~min}$ in $1.2 \%$ sodium hypochlorite and rinsed three times with sterile water. 50 wheat kernels were incubated in $10 \mathrm{ml}$ of a conidial suspension $\left(10^{4}\right.$ spores $\left.\mathrm{ml}^{-1}\right)$ of each F. proliferatum strain for $48 \mathrm{~h}$ on a shaker. Inoculated seeds were sowed in twice-autoclaved soil in pots (10 kernels per pot, five replicates per strain). Un-inoculated controls were treated with sterile water. The experiment was carried out twice.

In a greenhouse, the temperature cycled between $26^{\circ} \mathrm{C}$ (from 0600 to 2200 ) and $22^{\circ} \mathrm{C}$ (from 2200 to 0600 ); light of intensity 4000 to 5000 lux was maintaining for $16 \mathrm{~h}$; relative humidity was $50 \%$. Plants in pots were watered every other day till the kernels were ripe. After two more weeks without watering, ears, first flag leaves, and the internodes between last node and ear were collected.

\section{Re-isolation of fungi from wheat plants}

The stems (between the ear and the last node of the inoculated plants which were fully asymptomatic) were cut into 2-3 cm sections, surface sterilized with $1.2 \%$ sodium hypochlorite for $3 \mathrm{~min}$ and rinsed three times in autoclaved distilled water. Stem sections were placed on Czapek-Dox-Iprodione-Dichloran (CZID) medium (Abildgren et al. 1987) and incubated at $25^{\circ} \mathrm{C}$. Un-inoculated controls were handled in the same way. Fungal growth was observed after 3-4 days. Taxonomic identity of the isolates was confirmed by PCR with primers PRO1/PRO2 specific for F. proliferatum (Mulè et al. 2004).

\section{Quantification of fungal DNA in wheat stems, flag leaves and kernels}

Wheat kernels, flag leaves and stems were dried for $48 \mathrm{~h}$ at $45^{\circ} \mathrm{C}$ and ground. Total DNA was extracted from 50 mg using a CTAB method (Brandfass and Karlovsky 2008). The pellet was dissolved in $50 \mu \mathrm{l}$ TE buffer (10 mM Tris/HCl, $1 \mathrm{mM}$ EDTA, pH 8.0). DNA solution was diluted fifty fold prior to PCR analysis. Inhibition assay was carried out to confirm the absence of matrix effects on PCR at this dilution. F. proliferatum DNA in plant tissue was quantified as described previously (Nutz et al. 2011). The lowest standard set as a limit of quantification corresponded to DNA content of $0.935 \mathrm{ng} \mathrm{g}^{-1}$.

\section{Mycotoxin analysis in kernels}

$100 \mathrm{mg}$ of ground kernels were extracted with acetonitrile/water (84/16, v/v) as described (Adejumo et al. 2007); samples for beauvericin analysis were not defatted. HPLC separation and MS/MS detection was performed essentially as described (Adejumo et al. 2007) but ion trap 500-MS (Varian, Darmstadt, Germany) was used as a detector. Beauvericin was quantified in a positive ionization mode as a sodium adduct with mass transitions $\mathrm{m} / \mathrm{z} 806>645$ (quantification), $m / z 806>545$ and $m / z 806>384$ (confirmation). Calibration curves were constructed using analytical standards dissolved in methanol/water $(1: 1, \mathrm{v} / \mathrm{v})$ with a correction for recovery and matrix effects. The limit of quantification for both beauvericin and fumonisin $\mathrm{B}_{1}$ was $25 \mathrm{ng} \mathrm{g}^{-1}$.

\section{Statistical Analyses}

Statistical analyses were performed with SPSS 22.0 (IBM SPSS statistics, USA). The Pearson correlation coefficient (2-tailed) between mycotoxins (fumonisin $B_{1}$ and beauvericin) production and fungal DNA was calculated. 


\section{Results \\ F. proliferatum strains originating from different plants were capable of infecting wheat systemically}

We inoculated wheat seeds with conidia of F. proliferatum and sowed them in soil. The germination rate dropped from $94 \%$ for un-inoculated seeds to $84-92 \%$ for seeds inoculated with $F$. proliferatum. No disease symptoms were observed in the inoculated plants. To determine whether plants inoculated with $F$. proliferatum were colonized systemically, we attempted to re-isolate the pathogen and detect its DNA in stems, flag leaves and harvested kernels. Mycelia growing out from stems of infected wheat plants (Fig. 1a) were confirmed to belong to F. proliferatum by species-specific PCR (Fig. 1b). No mycelia grew out from surface-sterilized stems of un-inoculated plants.
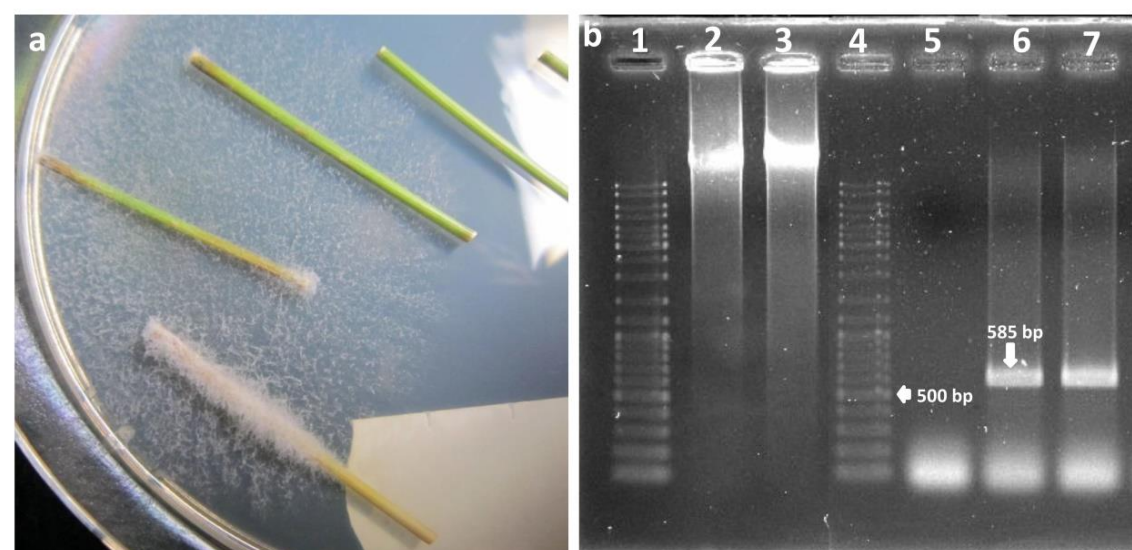

Fig. 1. Re-isolation of fungi from colonized wheat stems. (a) Fungal mycelia growing out from surface-sterilized wheat stem from seed-inoculated plants. (b) Two fungal colonies were randomly selected and fungal DNA was extracted (lanes 2 and 3). Assignment to F. proliferatum was confirmed by PCR (lanes 6 and 7). Lanes 1 and 4: DNA size standards; lane 5: negative control

The presence of $F$. proliferatum DNA in stems, flag leaves and harvested kernels corroborated systemic colonization (Fig. 2). F. proliferatum strains originating from different hosts successfully colonized wheat plants; the extent of colonization among strains from the same host plant differed. Strain F. proliferatum 29-M isolated from maize accumulated the highest amount of fungal DNA both in wheat stems and kernels.

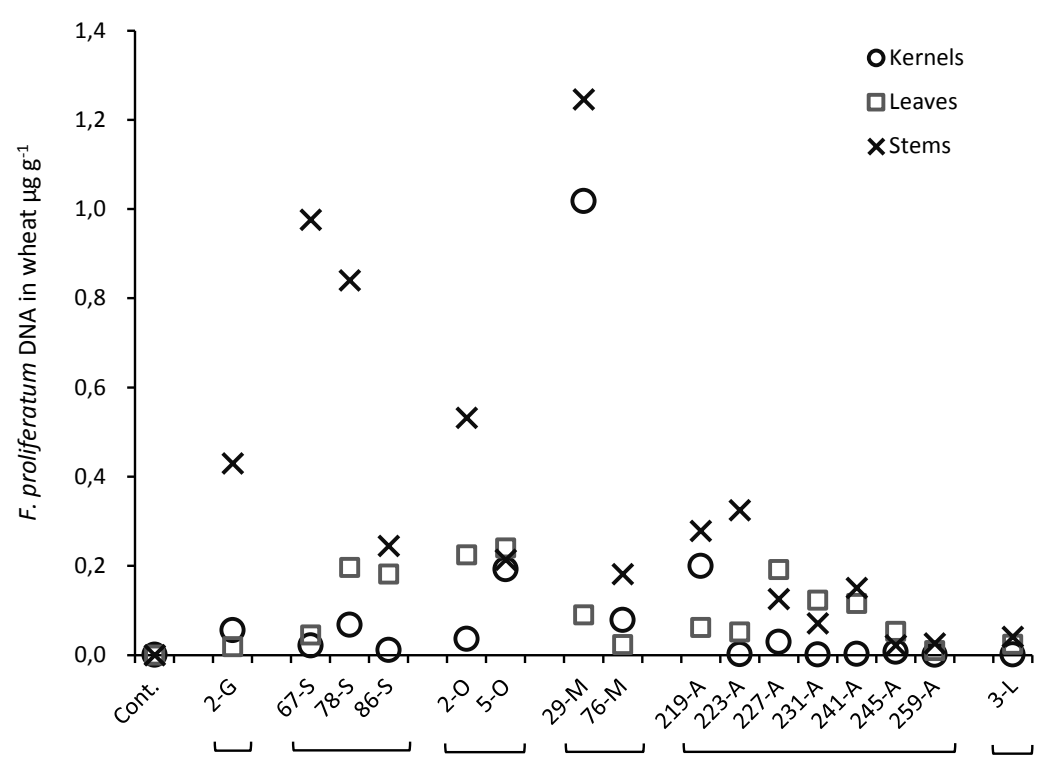

Fig. 2. Fungal DNA of $F$. proliferatum strains in wheat stems (the internode between the last node and ear), the first flag leaves, and kernels was quantified by real time PCR 
The amount of fungal DNA in kernels was lower than in stems and leaves (Fig. 2). The experiment was carried out for the second time with similar results (Fig. 3).

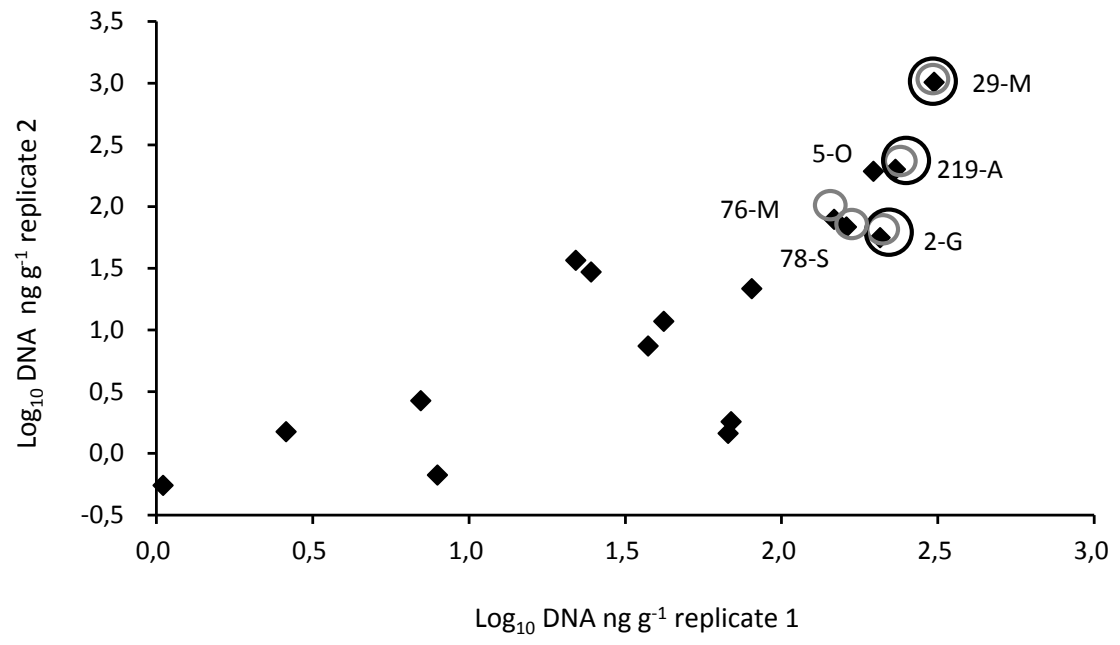

Fig. 3. Fungal DNA in kernels of wheat inoculated with $F$. proliferatum in two independent experiments. Samples encircled in gray contained beauvericin; samples encircled in black contained fumonisin $B_{1}$

\section{Fumonisin $\mathrm{B}_{1}$ and beauvericin accumulation}

Mycotoxins fumonisin $B_{1}$ and beauvericin were found in $1 / 3$ of infected wheat kernels (Fig. 4). The strains causing mycotoxin accumulation originated from different host plants.

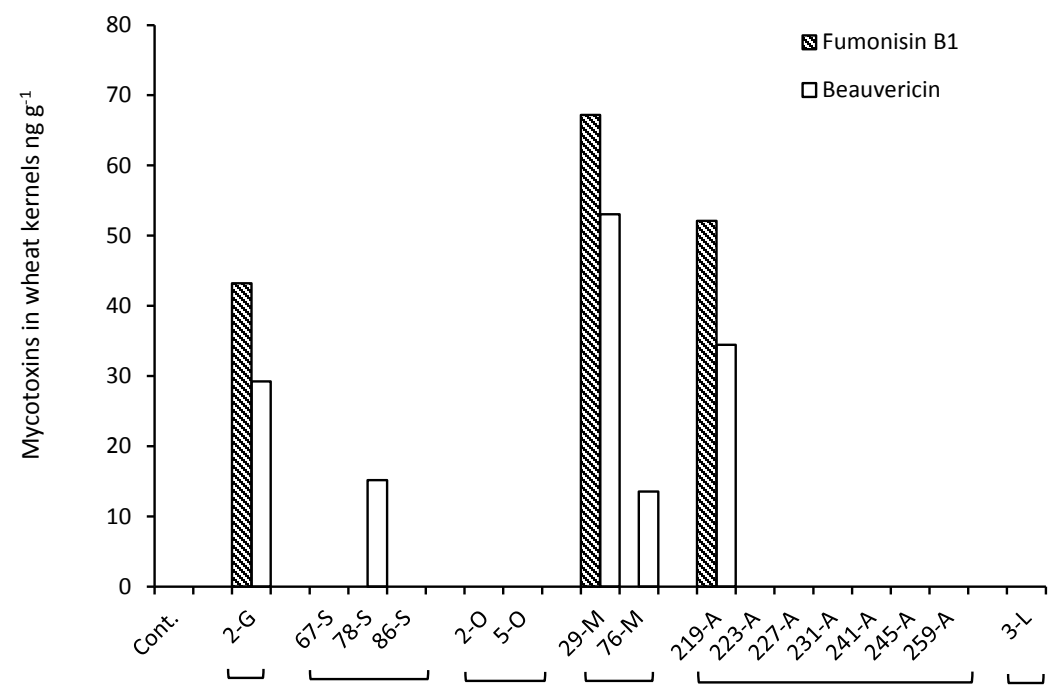

Fig. 4. Fumonisin $B_{1}$ and beauvericin quantification in colonized wheat kernels infected by different tested F. proliferatum strains with soak-inoculation mature seeds method

We found a strong positive correlation $(r=0.817)$ between DNA amount and fumonisin $B_{1}$ concentration in wheat kernels. A strong correlation ( $r=0.871$ ) was also found between F. proliferatum DNA and beauvericin content (Fig. 5). 


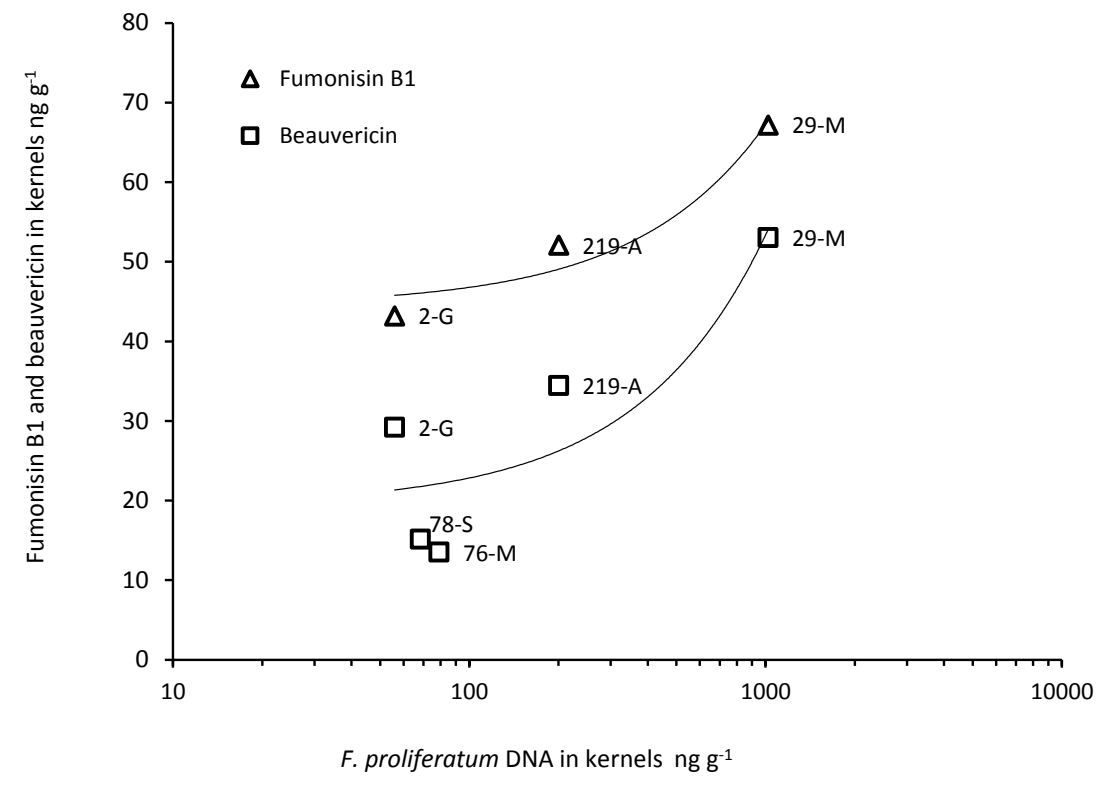

Fig. 5. Correlation of mycotoxin accumulation and amount of F. proliferatum DNA in wheat kernels

\section{Discussion}

F. proliferatum strains isolated from all host plants tested (garlic, onions, asparagus, maize, silver grass and dead larvae) were able to systemically infect wheat. The aggressiveness of the strains differed to a large extent. The limited number of strains used did not allow us to conclusively attribute these differences to the origin of the strains but low amount of biomass accumulated by all seven strains isolated from asparagus indicated that the specialization of $F$. proliferatum to asparagus may be accompanied by a decline of aggressiveness towards wheat. This might also be the case with onion and silver grass; more strains have to be tested to corroborate this hypothesis.

Only F. proliferatum strains that accumulated the largest amounts of DNA in kernels caused detectable contamination of kernels with mycotoxins (Fig. 3). Fumonisin $B_{1}$ was only detectable in kernels infected with three F. proliferatum strains out of sixteen strains tested. Interestingly, these three strains originated from different host plants: maize, asparagus, and garlic. Infection of wheat with two other strains resulted in the accumulation of beauvericin but not fumonisin $B_{1}$. The amounts of fumonisin $B_{1}$ in infected kernels were much lower than the levels commonly found in maize, supporting the hypothesis of Busman et al. (2012) that wheat kernels are a less favorable substrate for the production of fumonisins than maize. Fumonisin levels in naturally infected wheat were low, too (Desjardins et al. 2007, Palacios et al. 2011, Amato et al. 2015).

F. proliferatum DNA was found in largest amounts in stems, less in leaves and the lowest amounts in kernels. We speculate that $F$. proliferatum spreads within pith parenchyma (including the vascular system), which offers abundant nutrients and humidity, as shown for F. graminearum and F. culmorum (Guenther and Trail 2005, Mudge et al. 2006). Tight correlation between the amount of F. proliferatum DNA and mycotoxin concentration in kernels (Fig. 5 ) indicated that mycotoxins were produced in kernels rather than being transported to kernels from the stem with the transpiration stream. This speculation is in line with the results of Winter et al. (2013) on deoxynivalenol, which was produced in large amounts in stems of wheat plants infected with F. culmorum but did not reach kernels due to an anatomical barrier between grain and rachilla designated as "xylem discontinuity". Analysis of fumonisin and beauvericin content in stems of wheat plants infected with F. proliferatum via seeds will be needed to test this hypothesis. We have not observed any symptoms on the inoculated plants except that the leaves near the first node were more chlorotic as compared to control plants. Desjardins et al. (2007) reported kernel black points in wheat inoculated with F. proliferatum but they injected wheat spikelets at early anthesis while we studied seed-borne infection.

Accumulation of mycotoxins in symptomless wheat grain used for human and animal consumption poses a risk to human and animal health. Furthermore, residues of infected wheat plants can serve as source of inoculum of $F$. proliferatum for susceptible crops in the next season. Systemic infection of wheat with $F$. proliferatum therefore deserves attention, particularly in crop production systems in which seed coating with fungicides is not practiced. 


\section{Acknowledgements}

We would like to thank Prof. Dr. Carmen Büttner from Humboldt-Universität zu Berlin, Department of Crop and Animal Sciences, Division Phytomedicine for kindly providing the F. proliferatum strains. I wish to thank Yvonne Stelzer, Henrik Busch, Heike Rollwage for the technical assistance. This research was funded from the European Union Seventh Framework Programme (FP7/2007-2013) by "PlantFoodSec - Plant and Food Biosecurity" Project.

\section{References}

Abbas, H.K., Mirocha, C.J. \& Gunther, R. 1989. Mycotoxins produced by toxic Fusarium isolates obtained from agricultural and nonagricultural areas (Arctic) of Norway. Mycopathologia 105: 143-151.

Abbas, H.K., Cartwright, R.D., Xie, W., Mirocha, C.J., Richard, J.L., Dvorak, T.J., Sciumbato, G.L. \& Shier, W.T. 1999. Mycotoxin production by Fusarium proliferatum isolates from rice with Fusarium sheath rot disease. Mycopathologia 147: 97-104.

Abdalla, M.Y., Al-Rokibah, A., Moretti, A. \& Mule, G. 2000. Pathogenicity of toxigenic Fusarium proliferatum from date palm in Saudi Arabia. Plant Disease 84: 321-324.

Abildgren, M.P., Lund, F., Thrane, U. \& Elmholt, S. 1987. Czapek-Dox agar containing iprodione and dicloran as a selective medium for the isolation of Fusarium species. Letters in Applied Microbiology 5: 83-86.

Adejumo, T.O., Hettwer, U. \& Karlovsky, P. 2007. Survey of maize from south-western Nigeria for zearalenone, alpha- and betazearalenols, fumonisin $B_{1}$ and enniatins produced by Fusarium species. Food Additives and Contaminants 24: 993-1000.

Amato, B., Pfohl, K., Tonti, S., Nipoti, P., Dastjerdi, R., Pisi, A., Karlovsky, P. \& Prodi, A. 2015. Fusarium proliferatum and fumonisin B co-occur with Fusarium species causing Fusarium Head Blight in durum wheat in Italy. Journal of Applied Botany and Food Quality 88: 228-233.

Anonymous 2014. Beschreibende Sortenliste fuer Getreide, Mais, Ol- und Faserpflanzen, Leguminosen, Rueben, Zwischenfruechte 2013. Bundessortenamt, Hannover. $110 \mathrm{p}$.

Bacon, C.W. \& Hinton, D.M. 1996. Symptomless endophytic colonization of maize by Fusarium moniliforme. Canadian Journal of Botany 74: 1195-1202.

Bacon, C.W. \& Nelson, P.E. 1994. Fumonisin production in corn by toxigenic strains of Fusarium moniliforme and Fusarium proliferatum. Journal of Food Protection 57: 514-521.

Bai, G.-H. \& Shaner, G. 1996. Variation in Fusarium graminearum and cultivar resistance to wheat scab. Plant Disease 80: 975-979.

Bishop D.L., Levine H.G., Kropp B.R. \& Anderson A.J. 1997. Seedborne fungal contamination: Consequences in space-grown wheat. Phytopathology 87:1125-1133.

Brandfass, C. \& Karlovsky, P. 2008. Upscaled CTAB-based DNA extraction and real-time PCR assays for Fusarium culmorum and F. graminearum DNA in plant material with reduced sampling error. International Journal of Molecular Sciences 9: 2306-2321.

Busman, M., Desjardins, A.E. \& Proctor, R. 2012. Analysis of fumonisin contamination and the presence of Fusarium in wheat with kernel black point disease in the United States. Food Additives and Contaminants: Part A 29: 1092-1100.

Castella, G., Bragulat, M.R. \& Cabañes, F.J. 1999. Surveillance of fumonisins in maize-based feeds and cereals from Spain. Journal of Agricultural and Food Chemistry 47: 4707-4710.

Cirillo, T., Ritieni, A., Galvano, F. \& Cocchieri, R.A. 2003. Natural co-occurrence of deoxynivalenol and fumonisins $B_{1}$ and $B_{2}$ in Italian marketed foodstuffs. Food Additives \& Contaminats 20: 566-571.

Clear, R., \& Patrick, S. 1990. Fusarium species isolated from wheat samples containing tombstone (scab) kernels from Ontario, Manitoba, and Saskatchewan. Canadian Journal of Plant Science 70: 1057-1069.

Conner, R., Hwang, S. \& Stevens, R. 1996. Fusarium proliferatum: a new causal agent of black point in wheat. Canadian Journal of Plant Pathology 18: 419-423.

Dastjerdi, R. \& Karlovsky, P. 2015. Systemic infection of maize, sorghum, rice, and beet seedlings with fumonisin-producing and nonproducing Fusarium verticillioides strains. Plant Pathology Journal 31: 334-342.

Desjardins, A.E., Busman, M., Proctor, R.H. \& Stessman, R. 2007. Wheat kernel black point and fumonisin contamination by Fusarium proliferatum. Food Additives and Contaminants 24: 1131-1137.

Guenther, J.C. \& Trail, F. 2005. The development and differentiation of Gibberella zeae (anamorph: Fusarium graminearum) during colonization of wheat. Mycologia 97: 229-237.

Kwon, S-I, von Dohlen, C.D. \& Anderson, A.J. 2001. Gene sequence analysis of an opportunistic wheat pathogen, an isolate of Fusarium proliferatum. Canadian Journal of Botany 79: 1115-1121.

Leslie, J. F., Zeller, K. A., Logrieco, A., Mulè, G., Moretti, A. \& Ritieni, A. 2004. Species diversity of and toxin production by Gibberella fujikuroi species complex strains isolated from native prairie grasses in Kansas. Applied and Environmental Microbiology 70: 2254-2262.

Leslie, J.F., Summerell, B.A. \& Bullock, S. 2006. The Fusarium laboratory manual (Vol. 2): Blackwell publishing, Oxford. 388 p.

Logrieco, A., Moretti, A., Castella, G., Kostecki, M., Golinski, P., Ritieni, A. \& Chelkowski, J. 1998. Beauvericin Production by Fusarium Species. Applied and Environmental Microbiology 64: 3084-3088. 
Logrieco, A., Rizzo, A., Ferracane, R. \& Ritieni, A. 2002. Occurrence of beauvericin and enniatins in wheat affected by Fusarium avenaceum head blight. Applied and Environmental Microbiology 68: 82-85.

Marasas, W.F.O., Thiel, P.G., Rabie, C.J., Nelson, P.E. \& Toussoun, T. 1986. Moniliformin production in Fusarium section Liseola. Mycologia: 242-247.

Miller, J.D., Savard, M.E., Schaafsma, A.W., Seifert, K.A. \& Reid, L.M. 1995. Mycotoxin production by Fusarium moniliforme and Fusarium proliferatum from Ontario and occurrence of fumonisin in the 1993 corn crop. Canadian Journal of Plant Pathology 17: 233-239.

Moretti, A., Logrieco, A., Bottalico, A., Ritieni, A. \& Randazzo, G. 1994. Production of beauvericin by Fusarium proliferatum from maize in Italy. Mycotoxin Research 10: 73-78.

Mudge, A.M., Dill-Macky, R., Dong, Y., Gardiner, D.M., White, R.G. \& Manners, J.M. 2006. A role for the mycotoxin deoxynivalenol in stem colonisation during crown rot disease of wheat caused by Fusarium graminearum and Fusarium pseudograminearum. Physiological and Molecular Plant Pathology 69: 73-85.

Mulè, G., Susca, A., Stea, G. \& Moretti, A. 2004. A species-specific PCR assay based on the calmodulin partial gene for identification of Fusarium verticillioides, F. proliferatum and F. subglutinans. European Journal of Plant Pathology 110: 495-502.

Munkvold, G.P., McGee, D.C \& Carlton, W.M. 1997. Importance of different pathways for maize kernel infection by Fusarium moniliforme. Phytopathology 87: 209-217.

Nirenberg, H.I. \& O'Donnell, K. 1998. New Fusarium species and combinations within the Gibberella fujikuroi species complex. Mycologia: 434-458.

Nutz, S., Doll, K. \& Karlovsky, P. 2011. Determination of the LOQ in real-time PCR by receiver operating characteristic curve analysis: application to qPCR assays for Fusarium verticillioides and F. proliferatum. Analytical and Bioanalytical Chemistry 401: 717-726.

Osweiler, G.D., Ross, P.F., Wilson, T.M., Nelson, P.E., Witte, S.T., Carson, T.L., Rice, L.G. \& Nelson, H.A. 1992. Characterization of an epizootic of pulmonary edema in swine associated with fumonisin in corn screenings. Journal of Veterinary Diagnostic Investigation 4: 53-59.

Palacios, S.A., Ramirez, M.L., Cabrera Zalazar, M., Farnochi, M. C., Zappacosta, D., Chiacchiera, S.M., Reynoso, M.M., Chulze, S.N. \& Torres, A.M. 2011. Occurrence of Fusarium spp. and fumonisin in durum wheat grains. Journal of Agricultural and Food Chemistry 59: 12264-12269.

Placinta, C.M., D'mello, J.P.F. \& Macdonald, A.M.C. 1999. A review of worldwide contamination of cereal grains and animal feed with Fusarium mycotoxins. Animal Feed Science and Technology 78: 21-37.

Rheeder, J.P., Marasas, W.F.O., Theil, P.G., Sydenham, E.W., Shephard, G.S. \& van Schalkwyk, D.J. 1992. Fusarium moniliforme and fumonisins in corn in relation to human esophageal cancer in Transkei. Phytopathology 82: 353-357.

Riley, R.T., Wang, E., Schroeder, J.J., Smith, E.R., Plattner, R.D., Abbas, H., Yoo, H.S. \& Merrill, A.H. 1996. Evidence for disruption of sphingolipid metabolism as a contributing factor in the toxicity and carcinogenicity of fumonisins. Natural Toxins 4: 3-15.

Ritieni, A., Fogliano, V., Randazzo, G., Scarallo, A., Logrieco, A., Moretti, A., Manndina, L. \& Bottalico, A. 1995. Isolation and characterization of fusaproliferin, a new toxic metabolite from Fusarium proliferatum. Natural Toxins 3: 17-20.

Scott, P., Abbas, H., Mirocha, C., Lawrence, G. \& Weber, D. 1987. Formation of moniliformin by Fusarium sporotrichioides and Fusarium culmorum. Applied and Environmental Microbiology 53: 196-197.

Stankovic, S., Levic, J., Petrovic, T., Logrieco, A. \& Moretti, A. 2007. Pathogenicity and mycotoxin production by Fusarium proliferatum isolated from onion and garlic in Serbia. European Journal of Plant Pathology 118: 165-172.

von Bargen, S., Martinez, O., Schadock, I., Eisold, A.-M., Gossmann, M. \& Buttner, C. 2009. Genetic variability of phytopathogenic Fusarium proliferatum associated with crown rot in Asparagus officinalis. Journal of Phytopathology 157: 446-456.

Wilson, T.M., Ross, P.F., Rice, L.G., Osweiler, G.D., Nelson, H.A., Owens, D.L., Plattner, R.D., Reggiardo, C., Noon, T.H. \& Pickrell, J.W. 1990. Fumonisin B1 levels associated with an epizootic of equine leukoencephalomalacia. Journal of Veterinary Diagnostic Investigation 2: 213-216.

Winter, M., Koopmann, B., Doll, K., Karlovsky, P., Kropf, U., Schluter, K. \& von Tiedemann, A. 2013. Mechanisms regulating grain contamination with trichothecenes translocated from the stem base of wheat (Triticum aestivum) infected with Fusarium culmorum. Phytopathology 103: 682-689.

Zamani-Zadeh, H., \& Forutan, A. 1992. Isolation of Fusarium culmorum and F. proliferatum from wheat in Mazandaran. Iranian Journal of Plant Pathology 28:1-4. 